\title{
An Optimized Transmission Power Control Method for Pulsed BeiDou Pseudolite Signals
}

\author{
Di Wu*, Wei Chen and Anan Zhou
}

School of Automation, Wuhan University of Technology Wuhan, Hubei, China

\begin{abstract}
This paper presents an optimized transmission power control moethod for BeiDou pseudolites (BPLs) signals. Because of the near-far problem, the signal coverage probability of the conventional power transmission control method is nearly 50 percent at $95 \mathrm{dBm}$. Any further increase in transmission power only results in a decrease in the probability. In order to mitigate the near-far problem, a dynamic power control method was applied to transmit the signal. According to the previous parameter setting, we transmitted 3 pulses with a setp-to-step power reductionof $8 \mathrm{~dB}$. The simulation result shows that although the coverage probability is nearly 1.23 times higher, it suffers from a distinct decline when the distance exceeds $1.3 \mathrm{~km}$. Based on the BeiDou signal properties, we investigated the optimal parameter setting for the BPLs. It is proved by the assessment results that the optimal parameter setting is 2 pulses with a setp-to-step power reductionof $15 \mathrm{~dB}$. Compared with the previous setting, the optimized method delivers more stable signal coverage.
\end{abstract}

Keywords: BeiDou pseudolites, the near-far problem, signal coverage probability, transmission power control, pulsing scheme

\section{INTRODUCTION}

As one of the four main global navigation satellite systems (GNSS), the ChineseBeiDou satellite navigation system has been developed rapidly. A regional service has been initiated since December 2012 [1] with the capacity to support the Asian-Pacific users. By 2020 [2], the service will be upgraded to cover the entire world following the complete deployment of the satellite constellation. The system will provide two types of services: low precision open service and high precision authorized service, for users worldwide. The former refers to free service with an accuracy of $10 \mathrm{~m}$ for civilian users while the latter features higher levels of precision and integrity which are designed for authorized users [2].

However, due to the terrestrial complexity, some locations suffer from poor signal quality and service performance. In the worst case, such as in an urban canyon or a deep open mine, the receiver is unable to track the satellites because of the signal blockage [3]. A viable solution to this problem is deploying BeiDou pseudolites (BPLs) on the ground, which are BeiDou-like signal transmitters. Being a ground-based augmentation system, the primary benefit is that it can be used to compliment the BeiDou constellation if there are insufficient observable satellites. Moreover, by transmitting differential error corrections, the system is able to provide accuracy down to $10 \mathrm{~cm} \mathrm{[4].}$
On the other hand, the ground-based augmentation system suffers from severe near-far problem. The received satellite signal power is nearly constant, due to the huge distance from the satellite while the signal power from a BPL varies greatly in accordance with the distance from the user on the ground. At a certain distance, the received BPL signal power is strong enough to saturate the receiver and in fact disable the satellite signal detection [5]. Additionally, the signal coverage of the BPL is also limited by this problem [6].

Many techniques have been invented to mitigate the near-far problem. Among them, the most popular one, which is pulsing the signals [7], i.e., the Radio Technical Commission for Maritime Services (RTCM) SC-104 scheme [8] and the Radio Technical Commission for Aeronautics (RTCA) SC-159 scheme [9], because complex hardware modification at the receiver's end is not required. The basic idea of this method is to divide one period signal into several portions. Then, each portion is transmitted with high power, low duty cycle pulse which interferes with the satellite signals within merely a small part of the signal period. By delicately controlling the transmission power of the pulsed signals, it is possible to reduce the interference and enlarge the coverage probability for both GPS and GALILEO signals [10]. Because of different signal structures (i.e., code periods, signal strength, cross-correlation properties), it cannot be simply adopted to the BeiDou system without modifications. Therefore, anoptimized transmission power control method for BPL pulsed signals is proposed in this paper. This paper is organized as follows. In Section 2, the traditional power control method is described. Section 3 introduces the optimized parameter setting for the BPL transmission power control. The conclusion is made in Section 4. 


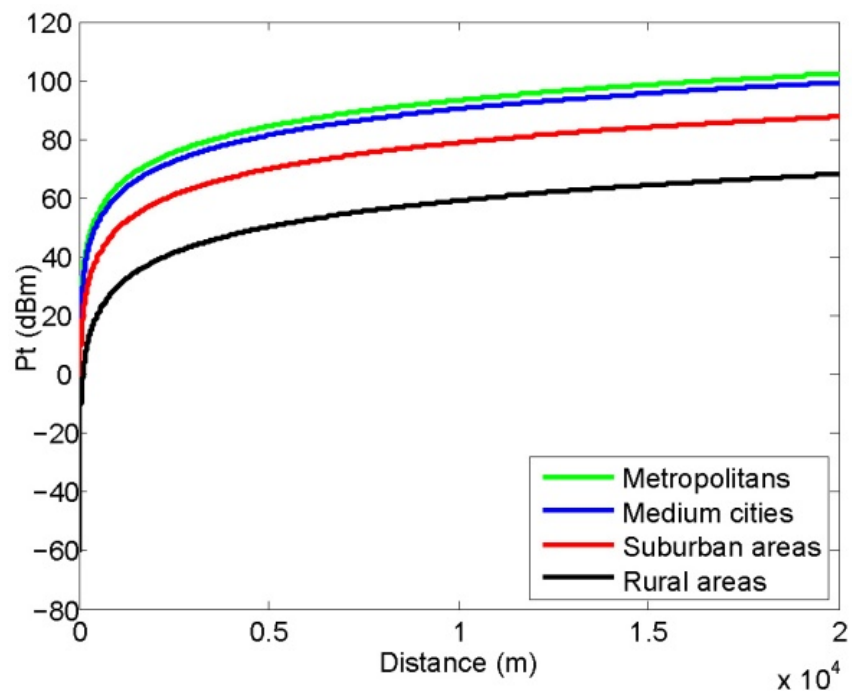

Fig. (1). BPL transmission power assumption using the COST-231 Hata model.

\section{TRADITIONAL TRANSMISSION POWER CON- TROL OF PULSED SIGNALS}

\section{A. Transmission Power Requirement}

Owing to the signal loss through the fading channel, a minimum transmission power is required to maintain the signal coverage within the desired area. To ensure the signal tracking at the far boundary, it is expected that the received pulsed signal power is the same as the nominal satellite power at $-133 \mathrm{dBm}-133 \mathrm{dBm}$, then the transmission power requirement considering the distance between the transmitter and the far boundary is expressed as [11]:

$$
P_{\mathrm{t}}=-133 \mathrm{dBm}+P_{\text {Puls }}+P_{\text {Prop }}
$$

where $\mathrm{P}_{\text {Puls Puls }}$ denotes the power loss caused by the signal pulsing (Puls $=20 \log ($ duty cycle) $)$, and $\mathrm{P}_{\text {Puls Prop }}$ represents the pass loss. The exact calculation of the path loss is impossible for the case of BPL signal transmission which complies to land mobile wireless communication channels which are extremely random and difficultto measure. In practical cases, the path loss is calculated in a statistical fashion.

Statistical methods are based on the pass losses collected from long-term field survey. Among the most commonly used methods, the COST-231 Hata model [12] is suitable for the pass loss prediction of land mobile wireless communication systems. The model contains four formations with respect to different environments: metropolitans, medium cities, suburban areas and rural areas, in which the medium city formation is the basic formation:

$$
\begin{aligned}
& P_{\text {prop_med }}=46.3+33.9 \log f_{\mathrm{c}}-13.82 \log h_{\mathrm{b}}-a\left(h_{\mathrm{r}}\right) \\
& +\left[44.9-6.55 \log h_{\mathrm{b}}\right] \log d(2) \\
& a\left(h_{\mathrm{r}}\right)=(1.1 \log f-0.7) h_{\mathrm{r}}-(1.56 \log f-0.8)
\end{aligned}
$$

where $f_{\mathrm{c}} \mathrm{f}_{\mathrm{c}}$ is the carrier frequency, $\mathrm{h}_{\mathrm{b}}$ is base station's antenna height, $d$ is the radio link distance, $a\left(h_{r}\right)$ is the mobile station's antenna height correction factor where $h_{r}$ is mobile station antenna height, $C=3 \mathrm{~dB}$ is the correction factor for metropolitans. Suburban and rural areas are expressed in (4) and (5) respectively:

$$
\begin{aligned}
& P_{\text {prop_sub }}=P_{\text {prop_med }}-2\left[\log \left(\frac{f_{\mathrm{c}}}{28}\right)\right]^{2}-5.4 \\
& \mathrm{P} \text { prop_rur }=
\end{aligned}
$$$$
\text { P prop_med }-4.78\left(\log f_{c} \quad\right)^{2}-18.33 \log \quad \text { c }-40.98(6)
$$

(5)Fig. (1) depicts the BPL transmission power assumption using the COST-231 Hata model in four typical environments.

\section{B. Signal Coverage Probability}

Owing to the shadowing effects caused by the obstacles, such as buildings, vehicles and human bodies, the signal power is sometimes lower than the requirement.In this case,within the service area, which is affected by the near-far problem, cannot be completely guaranteed. Therefore, the practically received signal power at the distance $d_{1}$ is expressed as:

$$
\mathrm{P}_{\mathrm{r}}=\mathrm{P}_{\mathrm{t}}-\mathrm{P}_{\text {Puls }}-\mathrm{P}_{\text {Prop }}-\varnothing P_{\mathrm{r}}=P_{\mathrm{t}}-P_{\text {Puls }}-P_{\text {Prop }}-\phi(6)
$$

where $\varnothing$ is a zero-mean Gaussian random variable with variance $\sigma^{22}$ which describes the shadowing effects. Consequently, the coverage probability at the distance ${ }_{1}$ is $P_{\text {in }}\left(P_{\min }\right.$ $\left., P_{\max },{ }_{1}\right)=p\left\{P_{\mathrm{r}}\left(_{1}\right)>P_{\min } \mid P_{\mathrm{r}}\left(\mathrm{d}_{11}\right)<P_{\max }\right\}$ which denotes the probability that the received power is between $P_{\min }$ and $P_{\max }$.

$$
P_{\text {in }}\left(P_{\min }, P_{\max }, d_{1}\right)=Q\left(\frac{P_{\min }-\overline{P_{\mathrm{r}}\left(d_{1}\right)}}{\sigma}\right)
$$




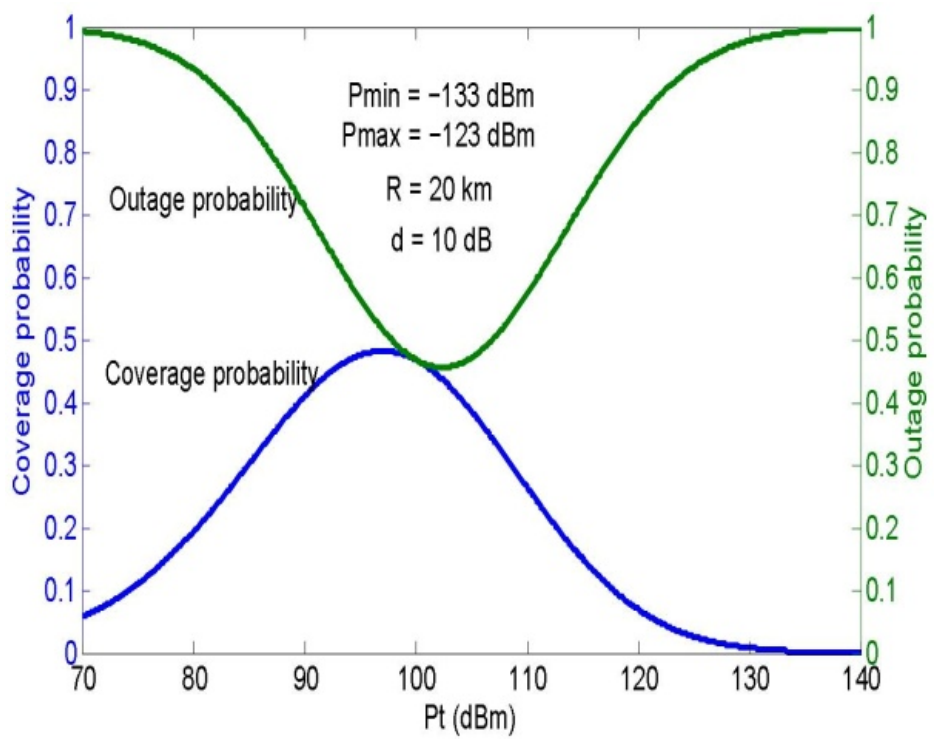

Fig. (2). Signal coverage probability considering the shadowing effects.

$$
-Q\left(\frac{P_{\max }-\overline{P_{\mathrm{r}}\left(d_{1}\right)}}{\sigma}\right)
$$

where $\overline{P_{\mathrm{r}}\left(d_{1}\right)}$ is the mean reception power at $\mathrm{d}_{11} \mathrm{~d}_{1}$. Then, (7) can be expressed in polar coordinates as:

$$
\begin{aligned}
& C=\frac{1}{\pi R^{2}} \int_{0}^{2 \pi} \int_{0}^{R} P_{\text {in }}\left(P_{\min }, P_{\max }, r\right) \cdot r d r d \theta \\
& =\frac{2}{R^{2}} \int_{0}^{R} r Q\left(a+b \log \frac{r}{R}\right) d r-\frac{2}{R^{2}} \int_{0}^{R} r Q\left(a+b \log \frac{r}{R}\right) d r \\
& =Q(a)+\exp \left(\frac{2-2 a_{\min } b}{b^{2}}\right) Q\left(\frac{2-a_{\min } b}{b}\right)-Q(a) \\
& -\exp \left(\frac{2-2 a_{\max } b}{b^{2}}\right) Q\left(\frac{2-a_{\max } b}{b}\right)
\end{aligned}
$$

where

The coverage probability in the working range is shown in Fig. (2). It is demonstrated that the maximum coverage probability is $50 \%$ with the optimal transmission power of $95 \mathrm{dBm}$. Any further increase in transmission power only results in a decrease in the probability, this is because of the near-far problem.

\section{AN OPTIMIZED TRANSMISSION POWER CON- TROL METHOD FOR BPL}

\section{A. Principle Of Dynamic Power Control method}

In order to increase the coverage probability which is constrained by the near-far problem, the dynamic transmission power control method is explained and illustrated in
Fig. (3). Assuming the code period is one, this method transmits multiple pulses with a step-by-step power reduction, other than the conventional method which transmits only one pulse. The cross-correlation margin $\mathrm{MdB} \mathrm{dB}$ determines the overall power reduction due to the near-far problem, each pulse is assigned with a power reduction of $\alpha=(\mathrm{M} /(\mathrm{N}-1)) \mathrm{dB} \mathrm{dB}$. Because the overall pulse duration $\tau_{1}+\tau_{2} \ldots \ldots+\tau_{n n}$ is not more than the maximum allowable duty cycle, the pulse number $\mathrm{N}$ is limited to

$1 \leq N \leq \frac{\text { the maximum duty cycle }}{\text { the minimum duty cycle }}$.

The transmission power control scheme is organized as follows: the first pulse is sent at the power $P_{1} \mathrm{dBmP} \mathrm{P}_{1} \mathrm{dBm}$ in the duration $\tau_{1}$ with a radius $R_{1}$, the second one is transmitted with a power reduction of $\alpha \alpha \mathrm{dBmdBm}$ in the duration $\tau_{2}$ to cover a radius $R_{2}$, the following $n-2$ pulses repeat the same procedure until the maximum allowable duty cycle. It is easy to prove that transmitting multiple pulses is capable of providing a wider service area, the total coverage probability can be obtained from (7):

$$
C_{\text {total }}=1-\left(1-C_{1}\right)\left(1-C_{2}\right) \cdots \cdot\left(1-C_{n}\right)
$$

An example is given to elaborate the effectiveness of the method. Suppose that there are 4 pulses transmitted one by one, the power reduction $\alpha=8 \mathrm{dBm}$ if the cross-correlation margin is $\mathrm{dB} \mathrm{dB}$ [13]. Because the maximum duty cycle is $10 \%$ of $\mathrm{m}$, the transmission time for each pulse is $\tau_{1}=\tau_{2}=$ $\tau_{3}=2.5 \%=\tau_{3}=2.5 \%$, while the coverage for each pulse $\mathrm{iR}_{1}=20 \mathrm{~km}, \mathrm{R}_{1}=10.82 \mathrm{~km}, \mathrm{R}_{1}=5.842 \mathrm{~km}, \mathrm{R}_{1}=3.131 \mathrm{~km}$. Fig. (4) shows the probability for each pulse as well as the overall probability. From Fig. (4) it can be concluded that the coverage is 1.13 times wider and the probability is 1.41 times higher.

However, parameter settings of this example are based on GPS signal characteristics, the probability suffers a sharp 


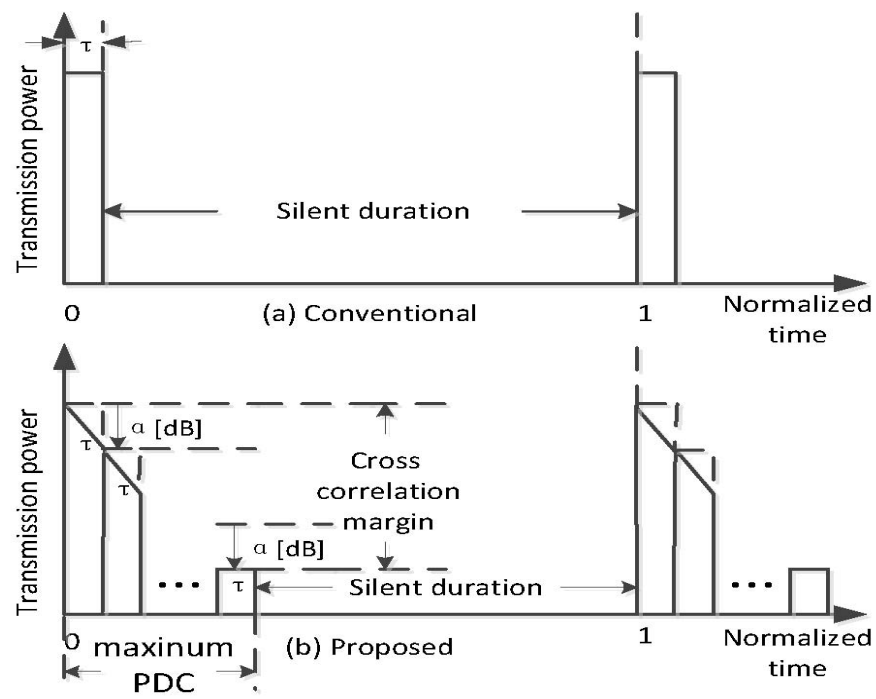

Fig. (3). Illustration of the dynamic power control method.

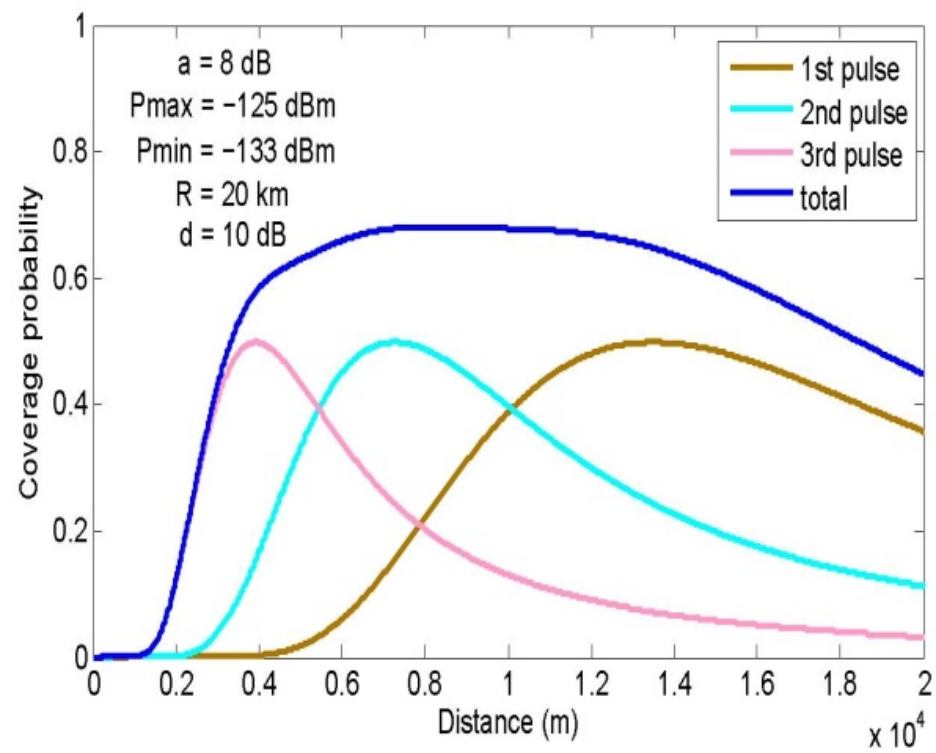

Fig. (4). Signal coverage probability for the GPS signal with the dynamic power control method.

decline if the distance exceeds $10 \mathrm{~km}$, for an optimized coverage, extensive discussion about optimal settings of $\alpha$ and $\mathrm{N}$ on the basis of the BPL code properties is carried out in the following chapters.

\section{B. An Optimal Parameter Setting For BPL}

According to [14-15], the worst-case cross-correlation margin with zero Doppler offset of the B1I code which is made of the sum of two 11-stage PRN code generators is 30 $\mathrm{dB}$, the maximum d must satisfy the situation of satellite tracking while the minimum dd must satisfy the situation of pseudolite tracking. Considering the most widely used 1 bit ADC convertor, the maximumdutycycle maximum duty cycle and the minimum duty cycle is $1 \%$ and $12 \%$, respectively. Therefore, the range of $\mathrm{N}$ is because the pulse number
$\mathrm{N}$ Nmust be an integer. Fig. (5) shows the total coverage probabilities of different $N(2 \sim 12)(2 \sim 12)$ in association with $\alpha=30 \mathrm{~dB} /(\mathrm{N}-1)$. It is noted that the probability reaches the maximum with $N=2, \alpha=15 \mathrm{~dB}$ for all environments. For medium cities, the probability is $0.77,1.13$ times bigger than the result shown in Fig. (5).

An assessment using the optimal parameter settings for four environments is carried out. The assessment results show that (Table 1) all environments are benefit from the proposed method. In the case of metropolitans (Fig. (6)), the maximum probability is 0.7260 , which is 1.4246 times higher than the traditional method, while the coverage is 1.0329 times wider. Moreover, it is clearly seen that the optimal parameter setting delivers better performance than the default setting. 


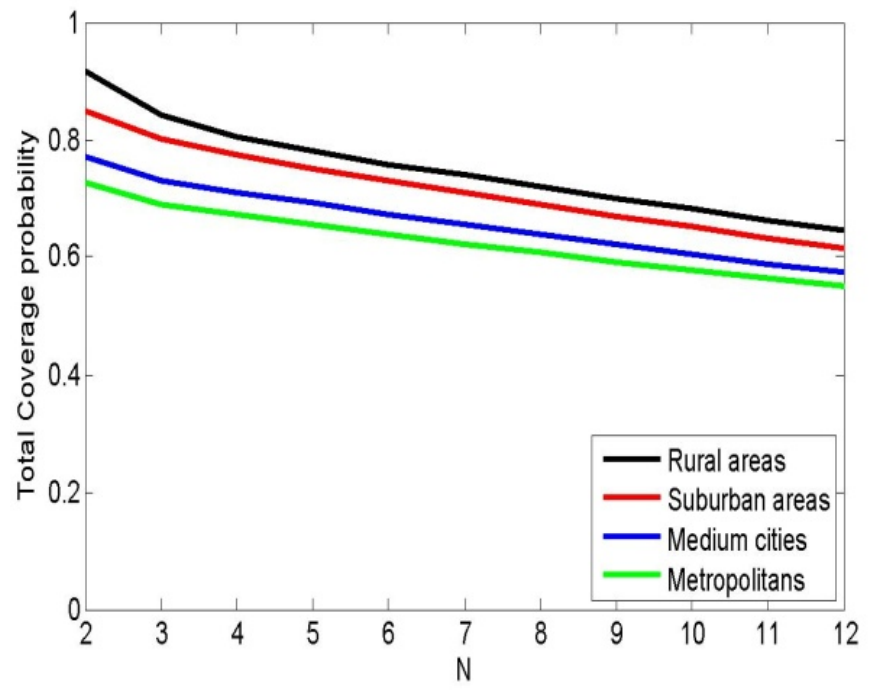

Fig. (5). Total coverage probability of pulse number $N(3 \sim 12)$.

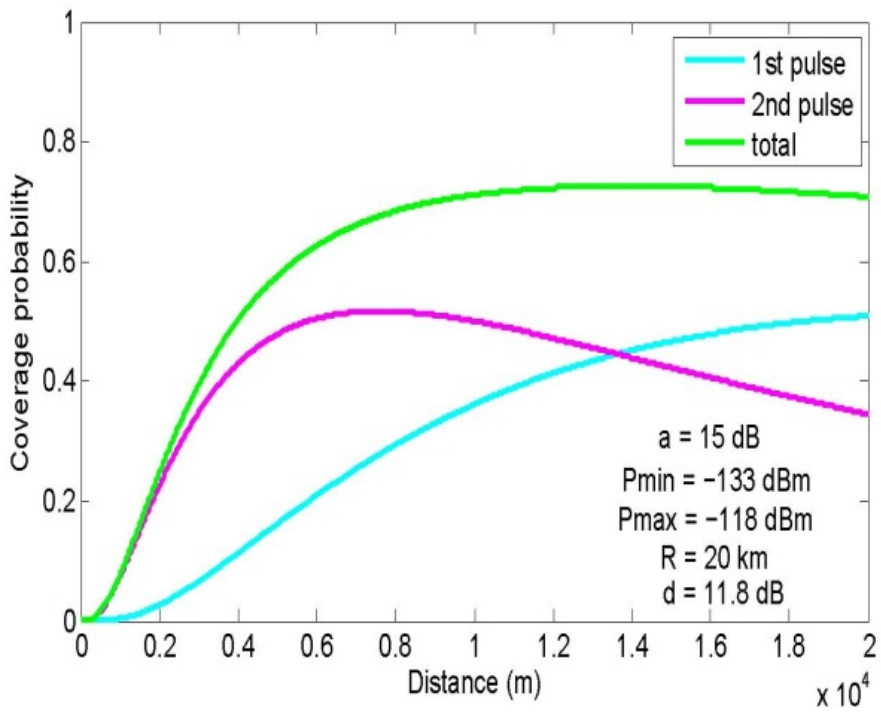

Fig. (6). Coverage probability for metropolitans with the optimal parameter setting.

Table 1. Assessment results of the simulation.

\begin{tabular}{|c|c|c|}
\hline Environment & Coverage Probability & Coverage Radius \\
\hline \hline Metropolitans & $142.46 \%$ & $103.29 \%$ \\
\hline Medium cities & $134.45 \%$ & $104.77 \%$ \\
\hline Suburban areas & $117.71 \%$ & $110.24 \%$ \\
\hline Rural areas & $109.99 \%$ & $116.35 \%$ \\
\hline
\end{tabular}

\section{CONCLUSION}

In this paper, a dynamic transmission power control method for the BPL is proposed which mitigates the near-far problem. The principle of the method is to use multiple pulses with a step-by-step power reduction to improve the coverage probability and radius. According to the BPL signal characteristics, different parameter settings are analyzed.
Simulation results shows that the best parameter setting is $N=2, \alpha=15 \mathrm{~dB}$ where the coverage probability of metropolitans is 1.4246 times higher and the coverage radius is 1.0329 times wider than the traditional method. It is also demonstrated that this method is able to deliver better performance for other environments.

\section{CONFLICT OF INTEREST}

The authors confirm that this article content has no conflicts of interest.

\section{ACKNOWLEDGEMENTS}

This work was financially supported by the National Natural Science Foundation of China (61074169), the National High Technology Research and Development Program of China (2013AA122403), and the Self-determined and Innovative Research Funds of WUT (2013-YB-018). 


\section{REFERENCES}

[1] China Satellite Navigation Office, "BeiDou Navigation Satellite System Open Service Performance Standard (Version 2.0)," Available from: http://www.BeiDou.gov.cn , 2015.

[2] O. Montenbruck, A. Hauschild, P. Steigenberger, U. Hugentobler, P. Teunissen, and S. Nakamura, "Initial Assessment of the COMPASS/BeiDou-2 Regional Navigation Satellite System," GPS Solutions, vol. 17, pp.211-222, 2013.

[3] H.H. Wang, C.R. Zhai, X.Q. Zhan, and Z. He, "Outdoor Navigation System Using Integrated GPS and Pseudolite Signals: Theoretical Analysis and simulation," IEEE International Conference on Information and Automation, pp.1127-1131, 2008.

[4] N. Awele, "GPS Pseudolite Signal Design. ION-GPS-94," Stanford University, Salt Lake City, Utah 1994.

[5] H.S. Cobb, GPSPseudolites: "Theory, Design, and Applications," PhD thesis, Stanford University 1997.

[6] S. Takahashi, "Transmission Power Control of Terrestrial Pseudo Satellite Signal for Global Navigation Satellite Systems," In: IEEE Tencon Region 10 Conference, pp.01-05, 2012.

A. Borio, and C. Odriscoll, "Design of a General Pseudolite Pulsing Scheme," IEEE Aerospace and Electronic Systems, vol. 50, pp. 2-16, 2014.

[7] T.A. Stansell, "RTCMCS-104 Recommended Pseudolite SignalSpecication," Global Positioning System, vol. 33, pp. 42-59, 1986.
[8] SC-159 RTCA, "GNSS based precision approach localarea augmentation system (LAAS) signal-in-spaceinterface control document (ICD),' Available from: http://www.rtca.org/index.asp, 2015.

[9] C.V. Bhaskar, K.J.S. Lorraine, and D.V. Ratnam, "Analysis of Near-Far Problem using Power Control Technique for GNSS based Applications," International Journal Of Engineering And Science, vol.4, pp. 01-08, 2014

[10] E.A. Lemaster, "Self-Calibrating, Pseudolite Arrays: Theory and Experiment," PhD Dissertation, Stanford University, 2002.

[11] N. Rakesh, B.U. Maheswari, and S.K. Srivatsa, "Performance Analysis of propagation models in different terrain conditions for IEEE Standard 802.16e WiMAX," In: International Conference on Communication and Signal Processing, pp.142-146, 2014.

[12] J.B.Y. Tsui, "Fundamentals of Global Positioning System Receivers: A Software Approach," John Wiley \& Sons, Inc., New York NY 2000.

[13] C.X. Li, J.P. Chen, and H.L. Chu, "Design of the pulse duty cycle of the pseudolite signal in local area augmentation systems," Science China Press and Springer-Verlag Berlin Heidelberg, vol. 54, pp. 1004-1008, 2011.

[14] B.W. Parkinson, and J.J. Spilker, "Global Positioning System: Theory and Applications," American Institute of Aeronautics and Astronautics, 1996.

[15] A. Goldsmith, "Wireless Communications," Cambridge University Press 2005.

(C) Wu et al.; Licensee Bentham Open.

This is an open access article licensed under the terms of the (https://creativecommons.org/licenses/by/4.0/legalcode), which permits unrestricted, non-commercial use, distribution and reproduction in any medium, provided the work is properly cited. 